\title{
SÍNDROME DE FRAGILIDAD EN EL ADULTO MAYORY LA SOBRECARGA DE SU CUIDADOR PRIMARIO
}

\section{FRAILTY SYNDROME IN THE ELDERLYAND OVERLOAD OF THEIR PRIMARY CAREGIVER}

Ma. de la Luz León-Vázquez ${ }^{*}$, Ma. Inés Medina-Rosete², Rosa I. Castellanos-Lima ${ }^{3}$, Zitlalli Portillo-García ${ }^{\text {, }}$ Rosalba Jaramillo-Sánchez y Alberto Limón-Aguilar ${ }^{5}$

${ }^{1}$ Consulta Externa Medicina Familiar, Hospital General de Subzona con Medicina Familiar 8, Instituto Mexicano del Seguro Social (IMSS); ${ }^{2}$ Unidad de Medicina Familiar 18, Admisión Médica Continua, IMSS, Santa Cruz; ${ }^{3}$ Consulta Externa Medicina Familiar, Hospital General de Zona 2, IMSS, Apizaco; ${ }^{4}$ Delegación Tlaxcala, IMSS; ${ }^{5}$ Consulta Externa Medicina Familiar. Unidad de Medicina Familiar 19, IMSS, Apizaco. Tlaxcala, México

RESUMEN: Introducción: El envejecimiento poblacional implica aumento de las demandas de atención sanitaria. La fragilidad es un paso previo a la discapacidad, que ocasiona un deterioro en la calidad de vida tanto en las personas que la padecen como en los encargados de su cuidado. Objetivo: Determinar la relación entre el síndrome de fragilidad en el adulto mayor con la sobrecarga del cuidador primario. Material y método: Estudio observacional, analítico. Incluyó 145 adultos mayores con cuidador primario, excluyéndose aquellos con deterioro cognitivo o discapacidad física severa. Previa autorización por el Comité Local de Investigación y firma de consentimiento, se interrogaron datos generales, se aplicó la escala Ensrud para evaluar fragilidad en el adulto mayor y la escala Zarit abreviada para sobrecarga del cuidador, en forma independiente. Se realizó un análisis descriptivo, chi cuadrada, U de Mann-Whitney, Kruskal-Wallis y correlación de Spearman, con el programa estadístico SPSS versión 22, considerando diferencia significativa un valor de $\mathrm{p}<0.05$. Resultados: El 61.4\% de los adultos mayores fueron mujeres, de edad $77 \pm 7$ años, el 59\% casados, el $70 \%$ con antecedente de enfermedad crónica degenerativa, el $28 \%$ se clasificaron como frágiles y el $50 \%$ como prefrágiles. El sexo, la edad, la escolaridad y las comorbilidades se correlacionaron con la presencia de fragilidad. El 70\% de los cuidadores fueron mujeres, de edad $52 \pm$ 15 años, el $64 \%$ casados, el $41 \%$ con sobrecarga intensa; ninguna variable se asoció con desgaste. Conclusión: El nivel de sobrecarga del cuidador tuvo una correlación positiva moderada con la fragilidad en el adulto mayor.

Palabras clave: Envejecimiento. Anciano frágil. Cuidadores.
ABSTRACT: Introduction: Population aging implies increased demands for care sanitary. The frailty of the elderly a previous step to disability, causing deterioration in the quality of life both in people who suffer, as in the caregivers. Objective: Determine the relationship between frailty syndrome in the elderly with the overload primary caregiver. Material and method: Analytical Observational study design. It included 145 older adults with a primary caregiver, excluding those with cognitive impairment or severe physical disability. After signing the consent, general data were interrogated; the Ensrud scales for frailty in the elderly and abbreviated Zarit scales for caregiver overload were applied independently. Descriptive analysis, chi square, Mann-Whitney and Spearman correlation, obtained with SPSS version 22 program, was considered significant difference with $\mathrm{p}<0.05$. Results: $61.4 \%$ of the older adults are women, age $77 \pm 7$ years, $59 \%$ married, $28 \%$ were classified as fragile and $50 \%$ as pre-fragile. Sex, age, schooling and comorbidities were associated with frailty. $70 \%$ of caregivers are women, $41 \%$ have have intense overload, and caregiver wear was associated within the elderly. Conclusion: The level of caregiver attrition had a moderate positive correlation with frailty in the elderly.

Key words: Aging. Frail elderly. Caregivers.
Correspondencia:

*Ma. de la Luz León-Vázquez

E-mail: clarissa023@gmail.com
Fecha de recepción: 31-01-2020

Fecha de aceptación: 20-05-2020
Disponible en internet: 31-07-2020

Rev Mex Med Fam. 2020;7:72-7

DOI: 10.24875/RMF.20000045

2007-9710 / @ 2020 Federación Mexicana de Especialistas y Residentes en Medicina Familiar. Publicado por Permanyer. Este es un artículo open access bajo la licencia CC BY-NC-ND (http://creativecommons.org/licenses/by-nc-nd/4.0/). 


\section{INTRODUCCIÓN}

El envejecimiento poblacional es uno de los mayores retos. A medida que envejecemos y dependiendo de los antecedentes físicos, psicológicos y sociales existen cambios en la fuerza vital y estilo de vida con una disminución en el estado de salud; una interacción entre estos factores se ha relacionado con la presencia de fragilidad $^{1}$, un factor predisponente a la discapacidad y deterioro en la calidad de vida tanto del adulto mayor como de los que están a su cuidado.

Enfermedades crónicas como la hipertensión arterial (HTA), la diabetes mellitus (DM2), la artritis, el cáncer y la enfermedad pulmonar obstructiva crónica se han reportado como predictores de la fragilidad ${ }^{2}$, y esta a su vez incrementa el riesgo de muerte ${ }^{3}$, por lo que en las últimas décadas se ha incrementado el interés por la fragilidad en el paciente geriátrico.

La fragilidad se considera un síndrome clínico geriátrico, de etiología multifactorial, el cual se caracteriza por la disminución de la fuerza, resistencia y funciones fisiológicas, lo que contribuye a su dependencia y/o muerte. La prevalencia de la fragilidad entre la población de 65 años y más varía entre el 7 y el $10 \%{ }^{1-5}$.

Se han descrito varios factores de riesgo, entre los que se encuentran: edad, sexo femenino, baja escolaridad, ingresos familiares bajos, mala autopercepción de salud, enfermedades cronicodegenerativas, tabaquismo, polifarmacia, hospitalización reciente, dos o más caídas en los últimos doce meses, fractura de cadera después de los 55 años y deterioro cognoscitivo ${ }^{6}$.

No presenta un único curso clínico, puede iniciar con diferentes síntomas en función de la causa desencadenante, encontrando datos no específicos (fatiga extrema, pérdida de peso inexplicable, infecciones frecuentes), caídas, deterioro del equilibrio y marcha, delirio e incapacidad fluctuante (inestabilidad diaria, días independientes y días en que requiere atención $)^{7}$. Las personas frágiles necesitan una persona que las cuide para satisfacer sus necesidades cotidianas, en ocasiones las 24 horas del día; este rol de cuidador recae en un miembro de la familia, quien debe hacerse responsable de su paciente (cuidador primario) ${ }^{7}$. Independientemente de si este rol es tomado como deber (trabajo) o como responsabilidad (por ser parte del núcleo familiar), el cuidador tiene una sobrecarga física, emocional, social y económica que conduce a mayor riesgo para su salud ${ }^{8,9}$, lo que se ha denominado síndrome de carga del cuidador (tomado del inglés burden, que traducido es «estar quemado»), descrito en 1974 por Freudenberguer ${ }^{10,11}$.

La carga del cuidador inicia cuando una persona adquiere funciones de cuidar a otra. Son varios los trastornos que se correlacionan con la carga del cuidador, incluyendo neurastenia, trastorno mixto ansioso depresivo y trastorno de adaptación ${ }^{11}$. El adulto mayor frágil y su cuidador cobran la misma importancia en el sistema de salud, siendo necesario identificarlos para poder dirigir una intervención oportuna a fin de mejorar su calidad de vida y evitar una progresión rápida y desenlace fatal. El objetivo del presente estudio fue determinar la relación que existe del síndrome de fragilidad en el adulto mayor con el desgaste del cuidador primario.

\section{MÉTODOS}

Se realizó un estudio observacional, transversal, analítico, de un total de 507 adultos mayores adscritos a la unidad. Se calculó una muestra de 145 adultos mayores y sus cuidadores primarios con base en el total de población de este grupo de edad, con un nivel de confianza de 0.95, en la Unidad Médica Familiar 18, IMSS Santacruz, Tlaxcala. Los participantes fueron elegidos por muestreo no aleatorio por conveniencia, sexo indistinto, que contaran con cuidador primario; se excluyeron aquellos con deterioro cognitivo o discapacidad física severa. Antes de participar firmaron el consentimiento informado. Se interrogaron datos generales (edad, sexo y escolaridad). Para evaluar 
la fragilidad en el adulto mayor se utilizaron los criterios de Ensrud (recomendados por la Guía de Práctica Clínica para población mexicana ${ }^{12}$ ), que consisten en valorar tres criterios: pérdida de al menos un $5 \%$ de peso independientemente de si fue de forma intencional o no en los últimos tres años, inhabilidad para levantarse de una silla cinco veces sin usar los brazos y nivel de energía reducida mediante la pregunta ¿ $_{i}$ Se siente usted lleno de energía?», considerando un «no», como respuesta. Si cumple con dos o tres criterios se considera anciano frágil, un criterio como prefrágil y ninguno como anciano robusto (considerando ningún criterio como robusto $)^{13}$. A los cuidadores se les aplicó una encuesta para datos generales (edad, sexo y escolaridad), además de la escala de Zarit (EZ) abreviada de cuidados paliativos para valorar el nivel de desgaste, que en comparación con las escalas reducidas se ha reportado como la que mejor se comporta en cuidadores principales de pacientes dependientes en atención primaria, con una sensibilidad del $100 \%$ y una especificidad del 90\%. La EZ abreviada consta de siete ítems y el punto de corte para diferenciar con y sin sobrecarga es de $17^{14,15}$. Las escalas, tanto para el paciente como para el cuidador, se aplicaron en forma independiente y aislada.

El análisis estadístico se llevó a cabo con el programa estadístico SPSS versión 22 , con estadística descriptiva univariante, con valoración de distribución mediante la prueba de Kolmogorov-Smirnov; para el análisis inferencial se utilizó la prueba de chi cuadrada $\left(\chi^{2}\right)$, Kruskal -Wallis y la correlación Spearman. Se consideraron diferencias significativas cuando el valor de $\mathrm{p}<0.05$. El protocolo fue presentado y autorizado por el Comité de Ética e Investigación del IMSS 2902 y se registró con folio R-2018-2902-007.

\section{RESULTADOS}

Se incluyeron 150 adultos mayores con cuidador primario, se eliminaron cinco encuestas por datos incompletos.

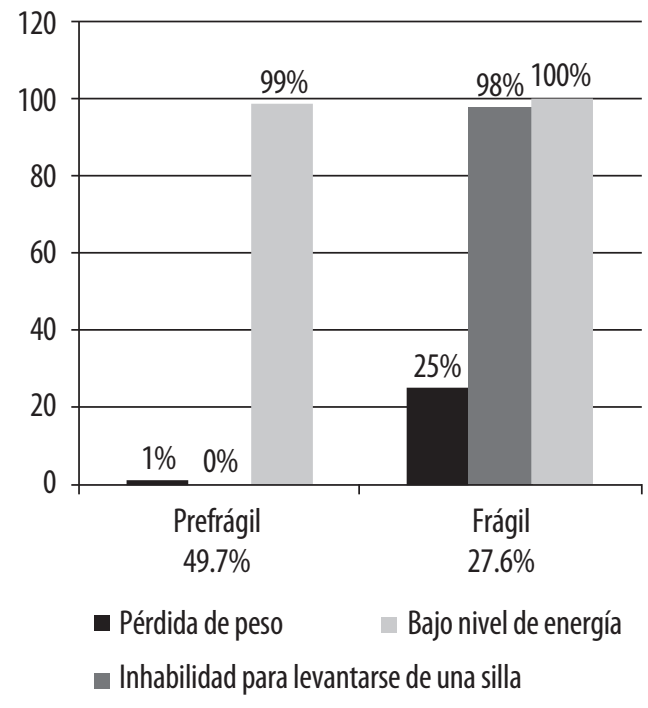

Figura 1. Fragilidad en el adulto mayor.

En cuanto a los adultos mayores: el $61.4 \%$ fueron mujeres, con edad promedio de $77 \pm 7$ años (76.3-78.6); el 58.6\% casados, el $7.6 \%$ separados y el $33.8 \%$ viudos; el $30 \%$ sin antecedentes de enfermedad cronicodegenerativa y el resto con antecedentes de enfermedades crónicas, principalmente HTA (40.6\%), DM (22\%), gonartrosis (4\%) y DM2 + HTA (0.7\%). Con base en la escala de Ensrud, el 27.6\% tuvieron fragilidad, el $49.7 \%$ se consideran prefrágiles y el $22.8 \%$ no tuvieron fragilidad. El nivel de energía se reportó como bajo en $76.6 \%$ (suma de prefrágil y frágil). ( Fig.1). la presencia de fragilidad en el adulto mayor estuvo estadísticamente asociada con la edad,estado civil, escolaridad y comorbilidades, no así el sexo (Tabla 1). La correlación de Spearman entre nivel de fragilidad y sobrecarga, fue baja (Tabla 2).

En relación con los cuidadores: el 69.7\% fueron de sexo femenino, la edad promedio fue $52 \pm 15$ años; el $63.4 \%$ casados, el $28.3 \%$ solteros y el $8.2 \%$ separados o viudos; el $38.6 \%$ con escolaridad primaria y el $21.4 \%$ con escolaridad secundaria. De acuerdo con la EZ abreviada, el 41.4\% tiene sobrecarga intensa. Ninguna variable del cuidador se asoció con la presencia de sobrecarga. 
Tabla 1. Fragilidad y características del adulto mayor y su asociación con el nivel de fragilidad

\begin{tabular}{|l|l|c|c|c|c|}
\hline \multicolumn{2}{|l|}{} & $\begin{array}{c}\text { Ninguno } \\
\mathbf{n}(\%)\end{array}$ & $\begin{array}{c}\text { Prefrágil } \\
\mathbf{n}(\%)\end{array}$ & $\begin{array}{c}\text { Frágil } \\
\mathbf{n}(\%)\end{array}$ & p \\
\hline \multirow{3}{*}{ Sexo } & Femenino & $21(63.6)$ & $45(62.5)$ & $23(57.5)$ & $0.834^{*}$ \\
\cline { 2 - 6 } & Masculino & $12(36.4)$ & $27(37.5)$ & $17(42.5)$ & \\
\hline \multirow{5}{*}{ Estado civil } & Viudo & $6(18.2)$ & $25(34.7)$ & $18(45)$ & $0.017^{*}$ \\
\cline { 2 - 6 } & Separado & $6(18.2)$ & $6(6.9)$ & 0 & \\
\cline { 2 - 6 } & Casado & $21(63.6)$ & $42(58.3)$ & $22(55)$ & \\
\cline { 2 - 6 } & Analfabeto & $4(12.1)$ & $6(8.3)$ & $8(20)$ & $0.021^{*}$ \\
\cline { 2 - 6 } & Primaria & $21(63.6)$ & $62(86.1)$ & $30(75)$ & \\
\cline { 2 - 6 } & Secundaria & $7(21.2)$ & $4(5.6)$ & $2(5)$ & \\
\cline { 2 - 6 } & Licenciatura & $1(3)$ & 0 & 0 & \\
\hline \multirow{5}{*}{ Comorbilidades } & Sin antecedente & $9(27.3)$ & $23(31.9)$ & $11(27.5)$ & $0.028^{*}$ \\
\cline { 2 - 6 } & HTA & $16(48.5)$ & $28(38.9)$ & $15(37.5)$ & \\
\cline { 2 - 6 } & DM2 & $8(24.2)$ & $18(25)$ & $6(15)$ & \\
\cline { 2 - 6 } & DM2 + HTA & 0 & $1(1.4)$ & 0 & \\
\cline { 2 - 6 } & Gonartrosis & 0 & $2(2.8)$ & $8(20)$ & \\
\hline \multirow{5}{*}{} & Edad (Media \pm DE) & $73 \pm 5$ & $77 \pm 6$ & $82 \pm 7$ & $0.000^{\dagger}$ \\
\cline { 2 - 6 } & Total & $\mathbf{3 3}$ & $\mathbf{7 2}$ & $\mathbf{4 0}$ & \\
\hline
\end{tabular}

*Prueba $\chi^{2}$.

${ }^{\top}$ Kruskal-Wallis.

HTA: hipertensión arterial; DM2: diabetes mellitus tipo 2.

Tabla 2. Sobrecarga del cuidador y fragilidad en el adulto mayor

\begin{tabular}{|l|c|c|c|c|}
\hline \multicolumn{5}{|c|}{ Sobrecarga de cuidador } \\
\cline { 1 - 3 } Fragilidad en adulto mayor & $\begin{array}{c}\text { Ausencia de sobrecarga } \\
\mathbf{n = 8 5}\end{array}$ & $\begin{array}{c}\text { Sobrecarga severa } \\
\mathbf{n = 6 0}\end{array}$ & Total & $\mathbf{p}$ \\
\hline Ninguno $\mathrm{n}(\%)$ & $27(31.8)$ & $6(10)$ & 33 & \\
\cline { 1 - 3 } Prefrágil $\mathrm{n}(\%)$ & $47(55.3)$ & $25(41.7)$ & 72 & \\
\cline { 1 - 4 } Frágil & $11(12.9)$ & $29(48.3)$ & 40 & 0.000 \\
\hline \multicolumn{4}{|c|}{$\chi^{2}$} & 0.000 \\
\hline
\end{tabular}

Se valoró la asociación entre el nivel de sobrecarga del cuidador y la fragilidad en el adulto mayor, encontrando diferencia significativa entre los grupos (Tabla 2), con una correlación de Spearman de 0.4, lo que denota una correlación moderada; el coeficiente de determinación es de 0.016 , por lo que se puede afirmar que la sobrecarga del cuidador se puede explicar en el $16 \%$ de los casos por la presencia de fragilidad en el adulto mayor.

\section{DISCUSIÓN}

La fragilidad en el adulto mayor, cada vez más frecuente, es una combinación de cambios relacionados con la edad y múltiples problemas médicos, coloca al paciente en una fase de transición entre un envejecimiento exitoso y la discapacidad ${ }^{16}$, e implica necesidad de cuidados especiales debido al aumento de las necesidades de asistencia sanitaria ${ }^{17}$. Se ha descrito una prevalencia 
de fragilidad variable en diferentes estudios, desde un 6 a un $98 \%$, esto en relación con el instrumento utilizado y edad, llegando a un $100 \%$ en mayores de 90 años ${ }^{18}$. En el presente estudio la prevalencia fue del $27.6 \%$, además de clasificar a casi la mitad de la población como prefrágiles, coincidiendo con lo reportado en estudios previos, en donde el estado prefrágil está presente en la mitad de la población geriátrica aun con diferente escala, lo que aumenta el efecto negativo en la salud de los pacientes ${ }^{19}$.

Con respecto a la escolaridad y la edad, se confirma su relación con la presencia de fragilidad descrita en la literatura ${ }^{19}$; aunque se ha reportado que las mujeres tienen mayor probabilidad de presentar fragilidad ${ }^{20,21}$, no encontramos diferencia significativa en relación con el sexo, probablemente por las características de la población estudiada (provenientes de un área suburbana).

Las características generales de los cuidadores primarios encontradas son similares a las de estudios previos, con predominio del sexo femenino, edad promedio de 52 años, casadas y con escolaridad primaria ${ }^{22-23}$.

El complejo rol de cuidador es asumido paulatinamente en la mayoría de las ocasiones sin formación o adiestramiento previo. Se reporta una relación significativa con el desarrollo de síntomas somáticos que disminuyen la calidad de vida de los cuidadores, repercutiendo en el cuidado del paciente a su cargo ${ }^{24}$.

La frecuencia de sobrecarga del cuidador es variable, algunos estudios reportan que más de la mitad de los cuidadores tiene algún grado de sobrecarga ${ }^{21,22}$. En la presente investigación se encontró una prevalencia de sobrecarga severa del cuidador del $41.4 \%$ (Tabla 2), otros estudios reportan porcentajes mayores (hasta más del $50 \%$ de los cuidadores) ${ }^{21,23-25}$, mientras que otros autores han encontrado frecuencia menor $(6.5 \%)^{26}$, lo que puede deberse al instrumento utilizado para medir la sobrecarga y el nivel de dependencia del adulto mayor, así como su edad y patología específica.
Coincidiendo con estudios anteriores, la presencia de fragilidad en el paciente estuvo en relación con el nivel de sobrecarga de su cuidador y las variables relacionadas con el paciente no presentaron asociación estadística $^{22,25}$.

En el presente estudio no se observó relación significativa entre la edad del cuidador y características tales como estado civil, el sexo y la escolaridad con la presencia de sobrecarga; coincidiendo con lo reportado en otros países ${ }^{21}$.

Existe controversia en cuanto a la relación entre el tiempo que lleva cuidando al paciente y el número de horas dedicadas al cuidado y la sobrecarga del cuidador, aunque es comprensible que a mayor tiempo empleado para el cuidado de otra persona haya más sobrecarga ${ }^{27,28}$.

Otro factor importante que considerar es el vínculo familiar con los pacientes. En su mayoría los cuidadores son familiares directos (pareja, hijos, nietos) y deben combinar el rol de cuidador con las responsabilidades del hogar y demandas conyugales y laborales, lo que interfiere en su calidad de vida y salud psicológica ${ }^{24}$, que en el presente estudio no se incluyeron como variables a estudiar. Los cuidadores formales (personas ajenas a la familia) también tienen un mayor nivel de estrés, sin embargo la sobrecarga observada es menor, aunque existe mayor predisposición al estrés ocupacional y al desgaste profesional o burnout ${ }^{29}$.

\section{CONCLUSIONES}

No se puede afirmar que la presencia de sobrecarga en el cuidador sea explicada por la fragilidad en el adulto mayor, ya que aunque existe correlación, esta es baja. Ello nos permite plantear una línea de investigación con búsqueda de posibles causas, además de proponer un programa de atención integral que incluya tanto al paciente como al cuidador, enfatizando en la capacitación y orientación a toda la familia para que el cuidado sea compartido, ya que el apoyo familiar es de vital importancia para el adulto mayor 
(no solo contar con esta red social, sino la percepción que tenga de esta) ${ }^{30}$.

Dentro de las limitaciones del estudio se encuentra el diseño transversal, ya que no podemos encontrar una relación causa-efecto, existiendo otras variables que pueden influir en la sobrecarga del cuidador, tales como relación familiar con el paciente, tiempo que llevan en ese rol y tiempo de dedicación a cuidar a su paciente, por lo que se recomendaría incluir todas estas variables en futuros estudios.

\section{FINANCIAMIENTO}

La presente investigación no ha recibido ayudas específicas provenientes de agencias del sector público, sector comercial o entidades sin ánimo de lucro .

\section{CONFLICTO DE INTERESES}

Los autores declaran no tener conflicto de intereses.

\section{RESPONSABILIDADES ÉTICAS}

Protección de personas y animales. Los autores declaran que para esta investigación no se han realizado experimentos en seres humanos ni en animales.

Confidencialidad de los datos. Los autores declaran que han seguido los protocolos de su centro de trabajo sobre la publicación de datos de pacientes.

Derecho a la privacidad y consentimiento informado. Los autores declaran que en este artículo no aparecen datos de pacientes.

\section{BIBLIOGRAFÍA}

1. Kojima G, Liljas-Ann EM, Iliffe S. Frailty syndrome: implications and challenges for health care policy. Risk Manag Healthc Policy. 2019;12:23-30.

2. Romero-Cabrera AJ. Perspectivas actuales en la asistencia sanitaria al adulto mayor. Rev Panam Salud Publica. 2008;24(4):288-94.

3. Escobar-Bravo MA, Jürschik P, Botigué T, Nuin C. La fragilidad como predictora de mortalidad en una cohorte de edad avanzada. Gac Sanit. 2014;28(6):489-91.

4. Carrazco-Peña K, Farías-Moreno K, Trujillo Hernández B. Frecuencia de envejecimiento exitoso y fragilidad. Factores de riesgo asociados. Rev Esp Geriatr Gerontol. 2017:53(1):23-5

5. Tello-Rodriguez T, Varela-Pinedo L. Fragilidad en el adulto mayor: detección, intervención en la comunidad y toma de decisiones en el manejo de enfermedades crónicas. Rev Peru Med Exp Salud Pública. 2016;33(2):238-34.

6. Acosta-Benito MA. Fragilidad en el anciano: del síndrome a la planificación sociosanitaria y legal. Rev Esp Med Legal. 2015:41(2):58-64.

7. Clegg A, Young J, Iliffe S, Rikkert MO, Rockwood K. Frailty in elderly people. Lancet. 2013;381(9868):752-62.
8. Ramón-Arbués E, Martínez-Abadía B, Martín-Gómez S. Factores determinantes de la sobrecarga del cuidador. Estudio de las diferencias de género. Aten Primaria. 2017:49(5):308-15.

9. Pérez-Cruz M, Muñóz-Martínez MA, Parra-Anguita L, Del PinoCasado R. Afrontamiento y carga subjetiva en cuidadores primarios de adultos mayores dependientes de Andalucía, España. Aten Primaria. 2017:49(7):381-8.

10. Aloe A, Amo L, Shanahan M. Classroom management self-efficacy and burnout: A multivariate meta-analysis. Educational Psychology Review [Internet]. 2014;26(1):101-26.

11. Vicente-Ruiz M, De la Cruz-García C, Morales-Navarrete RA, Martínez-Hernández CM, Villareal-Reyna MA. Cansancio, cuidados y repercusiones en cuidadores informales de adulto mayores con enfermedades crónicas degenerativas. Eur J Investig Health Psycho Educa. 2014;4(2):151-60.

12. Prevención, diagnóstico y tratamiento del síndrome de fragilidad en el anciano. Guía de Práctica clínica GPC IMSS-479-11. México: Instituto Mexicano del Seguro Social; 2011.

13. Ensrud K, Ewwing SK, Taylor BC, Fink HA, Cawthon PM, Stone K , et al. Comparison of 2 fraily index for prediction of fall, disability, fractures and death in older woman. Arch Int Med. 2008;168 (4):382-9.

14. Velez-Lopera J, Berbesi-Fernández D, Cardona Arango D, y cols. Validación de escalas abreviadas de Zarit para la medición de síndrome del cuidador primario del adulto mayor en Medellín. Aten Primaria. 2012;44(7):411-6.

15. Regueiro-Martinez MA, Pérez-Vázquez AP, Gómara-Villabona SM, Ferreiro-Cruz MC. Escala de Zarit reducida para la sobrecarga del cuidador en atención primaria. Aten Primaria. 2007:39(4):185-8

16. Tabue-Teguo M, Simo N, Gonzalez-Colaco HM, Cesari M, ÁvilaFunes JA, Féart $C$, et al. Frailty in elderly: a brief review. Geriatr Psychol Neuropsychiatr Vieil. 2017;15(2):127-37.

17. Gonzáles-Mechán MC, Leguía-Cerna J, Díaz-Vélez C. Prevalencia y factores asociados al síndrome de fragilidad en adultos mayores en la consulta de atención primaria de EsSalud, enero-abril 2015. Chiclayo, Perú. Revista Horizonte Médico [Internet]. 2017;17(3):35-42.

18. Rivadeneyra-Espinoza L, Sánchez-Hernández C. Síndrome de fragilidad en el adulto mayor en una comunidad rural de Puebla, México. Duazary. 2016;13(2):119-25.

19. Dos Santos-Tavares DM, De Freitas-Corrêa TA, Aparecida-Dias F Dos Santos-Ferreira PC, Sousa-Pegorari S. Frailty syndrome and socioeconomic and health characteristics among older adults. Colomb Med (Cali). 2017;48(3):126-31.

20. Pérez-Rodríguez M, Álvarez GómezT, Martínez Badaló EJ, Valdivia Cañizares S, Borroto Carpio I, Pedraza Núñez H. El síndrome de cuidador en cuidadores principales de ancianos con demencia Alzheimer. Gac Med Espirit [Internet]. 2017;19(1):38-50.

21. Zepeda-Alvarez PJ, Muñoz-Mendoza CL. Sobrecarga en cuidadores principales de adultos mayores con dependencia severa en atención primaria de salud. Gerokomos. 2019;30(1):2-5.

22. Delgado-Parada $E$, Suarez-Alvarez $O$, De Dios-Del Valle R, Valdespino-Páez I, Sousa-Ávila Y, Braña-Fernandez G. Características y factores relacionados con sobrecarga en una muestra de cuidadores principales de pacientes ancianos con demencia. Med Fam SEMERGEN. 2014;40(2):57-64.

23. Piñánez-García $M C$, Re-Domínguez ML, Núñez-Cantero $A M$ Sobrecarga en cuidadores principales de adultos mayores. Rev Salud Pública Parag. 2016;6(2):10-5

24. Romero Z, Cuba M. Síntomas somáticos en cuidadores de pacientes geriátricos con o sin sobrecarga, del área urbano-marginal Payet, Independencia, Lima, Perú. Rev Med Hered. 2013;24:204-9.

25. Flores G Elizabeth, Rivas R Edith, Seguel P Fredy. Nivel de sobrecarga en el desempeño del rol del cuidador familiar de adulto mayor con dependencia severa. Cienc Enferm. 2012;18(1):29-41.

26. Silva-Fhon JR, Gonzalez-Janampa JT, Mas-Huaman T, Marques $S_{\text {, }}$ Partezani-Rodriguez RA. Sobrecarga y calidad de vida del cuidador principal del adulto mayor. Av Enferm. 2016;34(3):251-8.

27. Segura-Dominguez N, Gómez-Caballo R, López-Quintana R, GilParedes E, Saiz-Cantero C, Cordero-Guevara J. El anciano dependiente y el desgaste físico y psíquico de su cuidador. Rev Esp Geriatr Gerontol. 2006;41(1):15-20.

28. Ávila-Tosacano JH, Vergara-Mercado M. Calidad de vida en cuidadores informales de personas con enfermedades crónicas. Aquichan. 2014;14(3):417-29.

29. Hernández-Zamora ZE, Ehrenzweig-Sánchez Y. Percepción de sobrecarga y nivel de burnout en cuidadores formales del adulto mayor institucionalizado. Enseñ Invest Psicol. 2008;13(1):127-42.

30. Morales-Cariño EM, Jiménez-Herrera BL, Serrano-Miranda TA. Evaluación del colapso del cuidador primario de pacientes adultos mayores con síndrome de inmovilidad. Rev Invest Clin. 2012;64(3):240-6. 